\title{
Algebraic independence of the values of generalized Mahler functions
}

\author{
by \\ Thomas TöPfer (Köln)
}

1. Introduction and results. In the last years arithmetic properties of holomorphic functions were studied which satisfy a functional equation of the shape

$$
P(z, f(z), f(T(z)))=0,
$$

where $P(z, u, w)$ is a polynomial with coefficients in $\overline{\mathbb{Q}}$, the field of all algebraic numbers, and $T(z)$ is an algebraic function. This generalizes investigations of Mahler [M1], [M2], [M3], which dealt with functional equations of the form

$$
f\left(z^{d}\right)=R(z, f(z))
$$

with $d \in \mathbb{N}, d \geq 2$, and a rational function $R(z, u)$ (resp. generalizations of these functional equations to several variables and several functions). Certain cases of (1) were studied extensively by different authors. For a survey of results about the transformations considered by Mahler see [M4], [K1], [L], [LP]. If $T(z)$ is a polynomial, the transcendence of $f(\alpha)$ for algebraic $\alpha$ was proved by Nishioka [Ni1]. This was generalized to algebraic functions $T(z)$ by Becker in [B3]. Applications to Böttcher functions were given by Becker and Bergweiler [BB], and transcendence measures for these functions can be found in [B4] (see also [NT]). The algebraic independence of several values $f_{1}(\alpha), \ldots, f_{m}(\alpha)$ was proved by Becker [B2] for certain rational transformations $T(z)$ under additional technical assumptions.

Since a general zero order estimate for functions satisfying (2) with $z^{d}$ replaced by rational functions $T(z)$ was proved in [T3], we will give an application of the zero order estimate in this paper and derive measures for the algebraic independence of the values of the functions considered by Becker in [B2]. Furthermore we give lower bounds for the transcendence degree of $\mathbb{Q}\left(f_{1}(\alpha), \ldots, f_{m}(\alpha)\right)$ over $\mathbb{Q}$, if $f_{1}, \ldots, f_{m}$ satisfy functional equations with more general rational transformations $T(z)$. 
TheOREM 1. Let $f_{1}, \ldots, f_{m}: U \rightarrow \mathbb{C}$ be holomorphic in a neighborhood $U$ of $\omega \in \widehat{\mathbb{C}}$, algebraically independent over $\mathbb{C}(z)$, and suppose the power series coefficients of $f_{1}, \ldots, f_{m}$ in the expansion at $\omega$ are algebraic. Suppose that $T(z)=T_{1}(z) / T_{2}(z)$ with $T_{1}, T_{2} \in \overline{\mathbb{Q}}[z], \operatorname{deg} T=\max \left\{\operatorname{deg} T_{1}, \operatorname{deg} T_{2}\right\}=$ $d \geq 2, \omega$ is a fixed point of $T$ of order $\operatorname{ord}_{\omega} T=d$, and $\underline{f}=\left(f_{1}, \ldots, f_{m}\right)$ satisfies the functional equation

$$
a(z) \underline{f}(z)=A(z) \underline{f}(T(z))+\underline{B}(z),
$$

where $A(z)$ is a regular $m \times m$ matrix with entries in $\overline{\mathbb{Q}}[z], \underline{B}(z) \in(\overline{\mathbb{Q}}[z])^{m}$, and $a(z) \in \overline{\mathbb{Q}}[z]$. Let $\alpha \in U$ be an algebraic number with $\lim _{k \rightarrow \infty} T^{k}(\alpha)=\omega$, where $T^{k}(\alpha)$ denotes the $k$-th iterate of $T$ at $\alpha$, and suppose for $k \in \mathbb{N}_{0}$ that $T^{k}(\alpha) \in U \backslash\{\omega, \infty\}$, and $T^{k}(\alpha)$ is neither a zero of a $(z)$ nor a zero of $\operatorname{det} A(z)$. Then for each polynomial $Q \in \mathbb{Z}\left[y_{1}, \ldots, y_{m}\right] \backslash\{0\}$ with $\operatorname{deg} Q \leq D$, where $\operatorname{deg} Q$ denotes the total degree of $Q$ in all variables, and $H(Q) \leq H$, where $H(Q)$ denotes the height of $Q$, i.e. the maximum of the moduli of the coefficients of $Q$, the inequality

$$
|Q(\underline{f}(\alpha))|>\exp \left(-c_{1} D^{m}\left(D^{m+2}+\log H\right)\right)
$$

holds with a constant $c_{1} \in \mathbb{R}_{+}$depending only on $\underline{f}$ and $\alpha$.

Remarks. (i) For $\omega=0, T(z)=p\left(z^{-1}\right)^{-1}$ with a polynomial $p \in \overline{\mathbb{Q}}[z]$, and a diagonal matrix $A(z)$, Theorem 1 is the quantitative analogue of the theorem in [B2], where the algebraic independence of the function values under consideration was proved.

(ii) With $T(z)=z^{d}, d \in \mathbb{N}, d \geq 2$, and $\omega=0$, Theorem 1 includes an earlier result of Becker (Theorem 1 in [B1]) and the improvement of Nishioka (Theorem 1 in [Ni2]).

TheOREM 2. Let $f_{1}, \ldots, f_{m}: U \rightarrow \mathbb{C}$ be holomorphic in a neighborhood $U$ of $\omega \in \widehat{\mathbb{C}}$, algebraically independent over $\mathbb{C}(z)$, and suppose the power series coefficients of $f_{1}, \ldots, f_{m}$ in the expansion at $\omega$ are algebraic. Suppose that $T(z)=T_{1}(z) / T_{2}(z)$ with $T_{1}, T_{2} \in \overline{\mathbb{Q}}[z], \operatorname{deg} T=d, \omega$ is a fixed point of $T$ with $\operatorname{ord}_{\omega} T=\delta \geq 2$, and $f=\left(f_{1}, \ldots, f_{m}\right)$ satisfies

$$
a(z) \underline{f}(z)=A(z) \underline{f}(T(z))+\underline{B}(z),
$$

where $A(z)$ is a regular $m \times m$ matrix with entries in $\overline{\mathbb{Q}}[z], \underline{B}(z) \in(\overline{\mathbb{Q}}[z])^{m}$, and $a(z) \in \overline{\mathbb{Q}}[z]$. Let $\alpha \in U$ be an algebraic number with $\lim _{k \rightarrow \infty} T^{k}(\alpha)=\omega$, and suppose for $k \in \mathbb{N}_{0}$ that $T^{k}(\alpha) \in U \backslash\{\omega, \infty\}$, and $T^{k}(\alpha)$ is neither a zero of a $(z)$ nor a zero of $\operatorname{det} A(z)$. Let $m_{0}$ be the greatest integer satisfying

$$
m_{0}<m\left(\frac{2 \log \delta}{\log d}-1\right)+\frac{\log \delta}{\log d} .
$$

Then

$$
\operatorname{trdeg}_{\mathbb{Q}} \mathbb{Q}(\underline{f}(\alpha)) \geq m_{0} .
$$


COROLlary 1. Suppose the assumptions of Theorem 2 are fulfilled with $d<\delta^{1+1 / 2 m}$. Then $f_{1}(\alpha), \ldots, f_{m}(\alpha)$ are algebraically independent. In particular, for $m=1$ and $d<\delta^{3 / 2}$ we have $f(\alpha) \notin \overline{\mathbb{Q}}$.

Remark. The case $m=1$ is Becker's result in [B3] in the special case of rational transformations and the functional equation (3).

TheOREM 3. Let $f_{1}, \ldots, f_{m}: U \rightarrow \mathbb{C}$ be holomorphic in a neighborhood $U$ of $\omega \in \mathbb{C}$, algebraically independent over $\mathbb{C}(z)$, and suppose $f_{1}(\omega), \ldots, f_{m}(\omega)$ are algebraic. Suppose that $T \in \overline{\mathbb{Q}}[z], \operatorname{deg} T=d, \omega$ is a fixed point of $T$ with $\operatorname{ord}_{\omega} T=\delta \geq 2$, and $\underline{f}=\left(f_{1}, \ldots, f_{m}\right)$ satisfies

$$
\underline{f}(z)=A(z) \underline{f}(T(z))+\underline{B}(z),
$$

where $A(z)$ is a regular $m \times m$ matrix with entries in $\overline{\mathbb{Q}}[z]$, and $\underline{B}(z) \in$ $(\overline{\mathbb{Q}}[z])^{m}$. Let $\alpha \in U$ be an algebraic number with $\lim _{k \rightarrow \infty} T^{k}(\alpha)=\omega$, and suppose for $k \in \mathbb{N}_{0}$ that $T^{k}(\alpha) \in U \backslash\{\omega\}$, and $\operatorname{det} A\left(T^{k}(\alpha)\right) \neq 0$. Let $m_{0}$ be the greatest integer satisfying

$$
m_{0}<(m+1) \frac{\log \delta}{\log d}
$$

Then

$$
\operatorname{trdeg}_{\mathbb{Q}} \mathbb{Q}(\underline{f}(\alpha)) \geq m_{0} .
$$

COROLlary 2. Suppose the assumptions of Theorem 3 are fulfilled and $d<\delta^{1+1 / m}$. Then $f_{1}(\alpha), \ldots, f_{m}(\alpha)$ are algebraically independent. In particular, for $m=1$ and $d<\delta^{2}$ we get $f(\alpha) \notin \overline{\mathbb{Q}}$.

Remark. Since the condition $d<\delta^{3 / 2}$ in Corollary 1 coincides with the condition given in the theorem of Becker in [B3] in the special case of rational transformations and functional equations of type (3), the weaker condition of Corollary 2 for polynomial transformations and the more restricted functional equations of type (4) gives a first answer to a question posed by Becker (p. 119 in [B3]). He asked for weaker technical assumptions of this form to extend the range of applications of Mahler's method.

2. Examples and applications. Our first example deals with series of the form

$$
\chi_{i}(z)=\sum_{h=0}^{\infty} q_{i}\left(T^{h}(z)\right) \quad(i=1, \ldots, m),
$$

where $T(z)=T_{1}(z) / T_{2}(z) \in \overline{\mathbb{Q}}(z), d_{j}=\operatorname{deg} T_{j}(j=1,2), \omega \in \mathbb{C}$ is a fixed point of $T$ of order $\delta \geq 2, q_{i} \in \overline{\mathbb{Q}}[z]$ with $\operatorname{deg} q_{i} \geq 1$ and $q_{i}(\omega)=0$ for $i=1, \ldots, m$. Then all $\chi_{i}$ are holomorphic in a neighborhood $U$ of $\omega$ and satisfy the functional equation

$$
\chi_{i}(z)=\chi_{i}(T(z))+q_{i}(z) \quad(i=1, \ldots, m) .
$$


Corollary 3. Suppose $q_{1}, \ldots, q_{m}$ are $\mathbb{C}$-linearly independent, $0<d_{2}<$ $d_{1}=d$, and $\alpha \in \overline{\mathbb{Q}}$ satisfies $\lim _{k \rightarrow \infty} T^{k}(\alpha)=\omega$ and $T^{k}(\alpha) \neq \omega$ for $k \in \mathbb{N}_{0}$. Then

$$
\operatorname{trdeg}_{\mathbb{Q}} \mathbb{Q}\left(\chi_{1}(\alpha), \ldots, \chi_{m}(\alpha)\right) \geq m_{0},
$$

where $m_{0}$ denotes the greatest integer satisfying

$$
m_{0}<(m+1) \frac{\log \delta}{\log d}-\left(1-\frac{\log \delta}{\log d}\right) m .
$$

Proof. For the application of Theorem 2 we have to show that $\chi_{1}, \ldots$ $\ldots, \chi_{m}$ are algebraically independent. In the next paragraph this will be derived from Lemma 6 of Section 3 .

Suppose that $\chi_{1}, \ldots, \chi_{m}$ are algebraically dependent. By Lemma 6 there exist $g_{i} \in \mathbb{C}(z)$ with $\operatorname{deg} g_{i}=\gamma_{i}(i=1,2), \gamma=\max \left\{\gamma_{1}, \gamma_{2}\right\}$, and $s_{1}, \ldots, s_{m} \in$ $\mathbb{C}$, not all zero, such that

$$
\frac{g_{1}(z)}{g_{2}(z)}=\frac{g_{1}(T(z))}{g_{2}(T(z))}+\sum_{i=1}^{m} s_{i} q_{i}(z) .
$$

Since the sum on the right is nonzero, we know that $\gamma \geq 1$. From this equation we get the polynomial identity

$$
g_{1}(z) h_{2}(z)=g_{2}(z) h_{1}(z)+g_{2}(z) h_{2}(z) \sum_{i=1}^{m} s_{i} q_{i}(z)
$$

with $h_{i}(z)=T_{2}(z)^{\gamma} g_{i}(T(z)) \in \mathbb{C}[z](i=1,2)$. Since $g_{1}, g_{2}$ resp. $T_{1}, T_{2}$ are coprime, we see that $h_{1}, h_{2}$ are also coprime. Thus $h_{2} \mid g_{2}$, and the condition $d_{2}<d_{1}$ implies

$$
\operatorname{deg} h_{2}=\left(\gamma-\gamma_{2}\right) d_{2}+\gamma_{2} d_{1} \leq \gamma_{2}=\operatorname{deg} g_{2} .
$$

But $d_{2} \geq 1, d_{1} \geq 2$ and $\gamma \geq 1$. Hence we get a contradiction, and so $\chi_{1}, \ldots, \chi_{m}$ must be algebraically independent. Then application of Theorem 2 completes the proof.

Corollary 4. Suppose that $1, q_{1}, \ldots, q_{m}$ are $\mathbb{C}$-linearly independent, $T(z) \in \overline{\mathbb{Q}}[z]$ with $2 \leq \delta \leq d, d \nmid \operatorname{deg}\left(\sum_{i=1}^{m} s_{i} q_{i}(z)\right)$ for arbitrary $\left(s_{1}, \ldots, s_{m}\right)$ $\in \mathbb{C}^{m} \backslash\{\underline{0}\}$, and $\alpha \in \overline{\mathbb{Q}}$ satisfies $\lim _{k \rightarrow \infty} T^{k}(\alpha)=\omega$ and $T^{k}(\alpha) \neq \omega$ for $k \in \mathbb{N}_{0}$. Then

$$
\operatorname{trdeg}_{\mathbb{Q}} \mathbb{Q}\left(\chi_{1}(\alpha), \ldots, \chi_{m}(\alpha)\right) \geq m_{0},
$$

where $m_{0}$ denotes the greatest integer satisfying

$$
m_{0}<(m+1) \frac{\log \delta}{\log d} .
$$

Proof. Under the assumption that $\chi_{1}, \ldots, \chi_{m}$ are algebraically dependent, we get analogously to the proof of Corollary 3 the polynomial identity 
(notice that $T_{2}=1$, hence $h_{2}=g_{2}$ )

$$
g_{1}(z) g_{2}(T(z))=g_{2}(z) g_{1}(T(z))+g_{2}(z) g_{2}(T(z)) \sum_{i=1}^{m} s_{i} q_{i}(z) .
$$

The coprimality of $g_{1}, g_{2}$ implies $g_{2}(T(z)) \mid g_{2}(z)$, hence $\gamma_{2}=0$. Now we compare the degrees in (5). The degree on the left side is $\gamma_{1}$, and the two terms on the right have degrees $\gamma_{1} d$ and $\operatorname{deg}\left(\sum_{i=1}^{m} s_{i} q_{i}(z)\right)=\Delta$, respectively. Since $d \geq 2$, this forces $\gamma_{1} d=\Delta$. But $\Delta$ is not divisible by $d$ except for $\Delta=0$. Then $\gamma_{1}=0$, and we get the contradiction $\sum_{i=1}^{m} s_{i} q_{i}(z)=0$. Therefore $\chi_{1}, \ldots, \chi_{m}$ are algebraically independent. Now application of Theorem 3 yields the assertion.

Corollary 5. Suppose $q_{1}, \ldots, q_{m}$ are $\mathbb{C}$-linearly independent, $T(z)=$ $T_{1}(z) / T_{2}(z) \in \overline{\mathbb{Q}}(z), 0<d_{2}<d_{1}=d=\delta$, and $\alpha \in \overline{\mathbb{Q}}$ satisfies $\lim _{k \rightarrow \infty} T^{k}(\alpha)$ $=\omega$ and $T^{k}(\alpha) \in U \backslash\{\omega\}$ for $k \in \mathbb{N}_{0}$. Then for each polynomial $Q \in \mathbb{Z}[y] \backslash\{0\}$ with $\operatorname{deg} Q \leq D$ and $H(Q) \leq H$,

$$
\left|Q\left(\chi_{1}(\alpha), \ldots, \chi_{m}(\alpha)\right)\right|>\exp \left(-c_{1} D^{m}\left(D^{m+2}+\log H\right)\right) .
$$

Proof. From the proof of Corollary 3 we know that $\chi_{1}, \ldots, \chi_{m}$ are algebraically independent. Since $\delta=d$, the assertion follows from Theorem 1.

Remark. The same quantitative result can be derived under the assumptions of Corollary 4 for $\delta=d$.

Now we consider certain Cantor series introduced by Tamura [Ta]. Let

$$
\theta_{i}(z)=\sum_{h=0}^{\infty} \frac{1}{q_{i}(z) q_{i}(T(z)) \ldots q_{i}\left(T^{h}(z)\right)} \quad(i=1, \ldots, m)
$$

with $T(z)=T_{1}(z) / T_{2}(z) \in \overline{\mathbb{Q}}(z), \operatorname{deg} T_{j}=d_{j}(j=1,2), \omega \in \widehat{\mathbb{C}}$ is a fixed point of $T$ of order $\delta \geq 2, q_{i} \in \overline{\mathbb{Q}}[z]$ with $\operatorname{deg} q_{i} \geq 1$ and $\left|q_{i}(\omega)\right|>1$ for $i=1, \ldots, m$ (notice that $\omega=\infty$ and $q_{i}(\infty)=\infty$ is possible). The functions $\theta_{i}$ are holomorphic in a neighborhood of $\omega \in \widehat{\mathbb{C}}$ and satisfy the functional equation

$$
\theta_{i}(T(z))=q_{i}(z) \theta_{i}(z)-1 \quad(i=1, \ldots, m) .
$$

Tamura proved the transcendence of $\theta(\alpha)$ for certain $\alpha$ in the special case $q(z)=z, T(z) \in \mathbb{Z}[z]$ and $\operatorname{deg} T \geq 3$. The more general case of polynomials $q_{i}, T \in \overline{\mathbb{Q}}[z](i=1, \ldots, m)$ was treated by Becker [B2]. He derived algebraic independence results for $\theta_{1}(\alpha), \ldots, \theta_{m}(\alpha)$ at algebraic points $\alpha$ and discussed in detail the transcendence of $\theta(\alpha)$ for linear polynomials $q$ and algebraic $\alpha$. Here we study rational transformations and give qualitative and quantitative generalizations of Becker's results. 
Corollary 6. Suppose $q_{1}, \ldots, q_{m}$ are pairwise distinct, $\max \left\{2, d_{2}\right\}<$ $d_{1}=d, 1 \leq \operatorname{deg} q_{i}<d-1$ for $i=1, \ldots, m$. Let $\alpha$ be an algebraic number with $\lim _{k \rightarrow \infty} T^{k}(\alpha)=\omega$ and $q_{i}\left(T^{k}(\alpha)\right) \neq 0, T^{k}(\alpha) \neq \omega$ for $k \in \mathbb{N}_{0}$ and $i=1, \ldots, m$. If $m_{0}$ is the greatest integer satisfying

$$
m_{0}<(m+1) \frac{\log \delta}{\log d}-\left(1-\frac{\log \delta}{\log d}\right) m
$$

then

$$
\operatorname{trdeg}_{\mathbb{Q}} \mathbb{Q}\left(\theta_{1}(\alpha), \ldots, \theta_{m}(\alpha)\right) \geq m_{0} .
$$

If $\delta=d$, then $\theta_{1}(\alpha), \ldots, \theta_{m}(\alpha)$ are algebraically independent, and for all polynomials $Q \in \mathbb{Z}[\underline{y}] \backslash\{0\}$ with $\operatorname{deg} Q \leq D$ and $H(Q) \leq H$,

$$
\left|Q\left(\theta_{1}(\alpha), \ldots, \theta_{m}(\alpha)\right)\right|>\exp \left(-c_{1} D^{m}\left(D^{m+2}+\log H\right)\right) .
$$

Proof. The assertions are obvious consequences of Theorems 1 and 2, if the algebraic independence of $\theta_{1}, \ldots, \theta_{m}$ is verified. Thus we assume that $\theta_{1}, \ldots, \theta_{m}$ are algebraically dependent, and apply Lemma 6 . First we must show that $q_{i}(z) / q_{j}(z)$ for $i \neq j$ is not of the form $g(T(z)) / g(z)$ for some $g \in$ $\mathbb{C}(z)$. With $g(z)=g_{1}(z) / g_{2}(z), \operatorname{deg} g_{i}=\gamma_{i}(i=1,2)$, and $\gamma=\max \left\{\gamma_{1}, \gamma_{2}\right\}$ we suppose on the contrary that

$$
q_{i}(z) g_{1}(z) h_{2}(z)=q_{j}(z) g_{2}(z) h_{1}(z)
$$

where $h_{i}(z)=T_{2}(z)^{\gamma} g_{i}(T(z)) \in \mathbb{C}[z]$. Since $g_{1}, g_{2}$ resp. $T_{1}, T_{2}$ are coprime, we see that $h_{1}, h_{2}$ are also coprime. Thus $h_{1}\left|q_{i} g_{1}, h_{2}\right| q_{j} g_{2}$, and this implies (notice that $d_{2}<d_{1}$ )

$$
\operatorname{deg} h_{i}=\gamma d_{2}+\gamma_{i}\left(d_{1}-d_{2}\right)=\gamma_{i} d_{1}+\left(\gamma-\gamma_{i}\right) d_{2} \leq d_{1}-2+\gamma_{i} \quad(i=1,2) .
$$

Since $d_{1} \geq 3$, we must have $\gamma_{1}=\gamma_{2}=0$, but this leads to the contradiction $q_{i}=q_{j}$. Now all conditions of Lemma 6 are fulfilled, and then there exist $i \in\{1, \ldots, m\}$ and a rational function $g$ (with $g_{i}, h_{i}, \gamma_{i}, \gamma$ as above) such that

$$
g_{2}(z) h_{1}(z)=h_{2}(z) g_{2}(z)+q_{i}(z) g_{1}(z) h_{2}(z) .
$$

Hence $h_{2} \mid g_{2}$, and this yields

$$
\operatorname{deg} h_{2}=\gamma_{2} d_{1}+\left(\gamma-\gamma_{2}\right) d_{2} \leq \gamma_{2} .
$$

But $d_{1} \geq 3$, and so $\gamma_{2}=d_{2}=0$. Now we compare the degrees on both sides of (7) and get $d_{1} \gamma_{1} \leq \gamma_{1}+d_{1}-2$. Since $d_{1} \geq 3$, we must have $\gamma_{1}=0$, but then $q_{i}(z)$ is a constant, and this is excluded. Thus $\theta_{1}, \ldots, \theta_{m}$ cannot be algebraically dependent.

Corollary 7. Suppose that $T \in \overline{\mathbb{Q}}[z]$ is a polynomial with $d \geq 2$, and $q \in \overline{\mathbb{Q}}[z]$ is a linear polynomial with $q(T(z))^{2} \neq q(z)^{2}-2$. Let $\alpha$ be an algebraic number with $\lim _{k \rightarrow \infty} T^{k}(\alpha)=\infty$ and $q\left(T^{k}(\alpha)\right) \neq 0$ for $k \in \mathbb{N}_{0}$. 
Then for each polynomial $Q \in \mathbb{Z}[y] \backslash\{0\}$ with $\operatorname{deg} Q \leq D, H(Q) \leq H$ the inequality

$$
|Q(\theta(\alpha))|>\exp \left(-c_{1} D\left(D^{3}+\log H\right)\right)
$$

holds for $\theta(z)$ as in (6). In particular, $\theta(\alpha)$ is an $S$-number in Mahler's classification of transcendental numbers.

P r o o f. In Corollary 2 of [B2] Becker showed that $\theta(z)$ is a transcendental function for $q(z), T(z)$ as above. Then Theorem 1 with $\omega=\infty$ yields the assertion (notice that $\operatorname{deg} T=d=\operatorname{ord}_{\infty} T$ ).

The next example deals with the series

$$
\Omega(z)=\sum_{h=0}^{\infty} \frac{(-1)^{h}}{q\left(T^{h}(z)\right)}
$$

with $q, T \in \overline{\mathbb{Q}}[z]$ and $\operatorname{deg} q \geq 1, d \geq 2$, which was introduced by Becker [B2]. Then $\Omega(z)$ is holomorphic in a neighborhood of $\omega=\infty$ and satisfies

$$
\Omega(T(z))=-\Omega(z)+1 / q(z) .
$$

Corollary 8. Suppose $q(T(z)) \neq \lambda^{-1} q(z)^{2}+q(z)-\lambda$ for any $\lambda \in \mathbb{C} \backslash\{0\}$, and $\alpha$ is an algebraic number with $\lim _{k \rightarrow \infty} T^{k}(\alpha)=\infty$ and $q\left(T^{k}(\alpha)\right) \neq 0$ for $k \in \mathbb{N}_{0}$. Then for each $Q \in \mathbb{Z}[y] \backslash\{0\}$ with $\operatorname{deg} Q \leq D$ and $H(Q) \leq H$,

$$
|Q(\Omega(\alpha))|>\exp \left(-c_{1} D\left(D^{3}+\log H\right)\right) .
$$

In particular, this transcendence measure is valid for Cahen's constant

$$
C=\sum_{h=0}^{\infty} \frac{(-1)^{h}}{S_{h}-1},
$$

where $S_{0}=2$ and $S_{h+1}=S_{h}^{2}-S_{h}+1$ for $h \geq 0$.

Remark. The transcendence of $C$ was proved by Davison and Shallit [DS] with continued fractions and later by Becker in [B2] using the identity $C=\Omega(2)$ for $q(z)=z-1, T(z)=z^{2}-z+1$. Corollary 8 implies that $C$ is a $S$-number in Mahler's classification of transcendental numbers.

Pro of of Corollary 8. In Corollary 3 of [B2] the transcendence of the function $\Omega(z)$ was proved. Then Theorem 1 yields the assertion.

The last example was studied by Becker in [B3], Corollary 1. Let

$$
\sigma(z)=\prod_{h=0}^{\infty} q\left(T^{h}(z)\right),
$$

where $q \in \overline{\mathbb{Q}}[z], \operatorname{deg} q \geq 1$, and $T(z)=T_{1}(z) / T_{2}(z) \in \overline{\mathbb{Q}}(z), \operatorname{deg} T_{i}=d_{i}$ $(i=1,2)$, and $\omega \in \widehat{\mathbb{C}}$ is a fixed point of $T$ of order $\delta$. Assume that $q(\omega)=1$. 
Then $\sigma(z)$ is holomorphic in a neighborhood of $\omega$ and satisfies the functional equation

$$
\sigma(z)=q(z) \sigma(T(z)) .
$$

Corollary 9. Suppose $0<d_{2}<d_{1}=\delta$, and $\alpha$ is an algebraic number with $\lim _{k \rightarrow \infty} T^{k}(\alpha)=\omega$ and $q\left(T^{k}(\alpha)\right) \neq 0, T^{k}(\alpha) \neq \omega, \infty$ for $k \in \mathbb{N}_{0}$. Then for any polynomial $Q \in \mathbb{Z}[y] \backslash\{0\}$ with $\operatorname{deg} Q \leq D, H(Q) \leq H$,

$$
|Q(\sigma(\alpha))|>\exp \left(-c_{1} D\left(D^{3}+\log H\right)\right) .
$$

Proof. The transcendence of $\sigma(z)$ was proved in Corollary 1 of [B3]. Then the assertion follows from Theorem 1.

3. Preliminaries and auxiliary results. Throughout the paper let $K$ denote an algebraic number field, and $O_{K}$ is the ring of integers in $K$. Define $\alpha$, the house of the algebraic number $\alpha$, as the maximum of the moduli of the conjugates of $\alpha$. A denominator of an algebraic number $\alpha$ is a positive integer $d$ such that $d \alpha \in O_{K}$. For a polynomial $P$ with algebraic coefficients the height $H(P)$ is defined as the maximum of the houses of the coefficients, and the length $L(P)$ is the sum of the houses of the coefficients.

LEMma 1. Suppose the rational function $g(z)=r(z) / s(z) \in K(z)$ is holomorphic in a neighborhood of $z=0$. Then for each $h \in \mathbb{N}_{0}$ the power series coefficients $g_{h}$ of

$$
g(z)=\sum_{h=0}^{\infty} g_{h} z^{h}
$$

satisfy

(i) $g_{h} \in K\left(g_{0}\right)$,

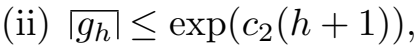

(iii) $D^{\left[c_{2}(h+1)\right]} g_{h} \in O_{K}$

with suitable $D \in \mathbb{N}$ and $c_{2} \in \mathbb{R}_{+}$depending only on $g$.

Proof. From $r(z)=s(z) \sum_{h=0}^{\infty} g_{h} z^{h}$ with $r(z)=\sum_{i=0}^{l} r_{i} z^{i}, s(z)=$ $\sum_{i=0}^{l} s_{i} z^{i}$ we get the following recurrence relation for the coefficients $g_{h}$ (with $r_{h}=0$ for $h>l$ ), $h \in \mathbb{N}_{0}$ :

$$
g_{h}=\frac{r_{h}}{s_{0}}-\sum_{\mu=1}^{\min \{l, h\}} \frac{s_{\mu}}{s_{0}} g_{h-\mu} .
$$

This implies the assertion. 
Lemma 2. Suppose $T(z)=T_{1}(z) / T_{2}(z)$ is a rational function with $\delta=$ $\operatorname{ord}_{0} T \geq 2$, and $\alpha \in \mathbb{C}$ satisfies $T^{k}(\alpha) \neq 0$ for $k \in \mathbb{N}_{0}$ and $\lim _{k \rightarrow \infty} T^{k}(\alpha)$ $=0$. Then for all $k \geq \bar{k}$,

$$
-c_{3} \delta^{k} \leq \log \left|T^{k}(\alpha)\right| \leq-c_{4} \delta^{k}
$$

with $c_{3}, c_{4} \in \mathbb{R}_{+}, \bar{k} \in \mathbb{N}$ depending on $T$ and $\alpha$.

Proof. Since 0 is a zero of $T$ of order $\delta \geq 2$, we have $T(z)=z^{\delta} g(z)$, where $g(z)$ is holomorphic in a neighborhood of $z=0$ and $g(0) \neq 0$. Then there exists a constant $\varepsilon \in \mathbb{R}_{+}$depending only on $T$ such that for all $\beta \in \mathbb{C}$ with $0<|\beta|<\varepsilon(<1)$,

$$
\gamma_{0}|\beta|^{\delta} \leq|T(\beta)| \leq \gamma_{1}|\beta|^{\delta},
$$

where $\gamma_{0}, \gamma_{1} \in \mathbb{R}_{+}$depend on $T$. Thus

$$
\exp \left(-\gamma_{2} \delta^{k}\right) \leq \gamma_{0}^{k}|\beta|^{\delta^{k}} \leq\left|T^{k}(\beta)\right| \leq \gamma_{1}^{k}|\beta|^{\delta^{k}} \leq \exp \left(-\gamma_{3} \delta^{k}\right)
$$

with $\gamma_{2}, \gamma_{3} \in \mathbb{R}_{+}$depending on $T$ and $\beta$. Since $\lim _{k \rightarrow \infty} T^{k}(\alpha)=0$, we know $0<\left|T^{k}(\alpha)\right|<\varepsilon$ for $k \geq \bar{k}$ with $\bar{k} \in \mathbb{N}$ depending on $T$ and $\alpha$, and together with (8) this yields the assertion.

The proofs of the theorems depend on the following results from elimination theory.

Lemma 3. Suppose $\underline{\omega} \in \mathbb{C}^{m}$. Then there exists a constant $c_{5}=c_{5}(\underline{\omega}, K)$ $\in \mathbb{R}_{+}$with the following property: If there exist increasing functions $\Psi_{1}, \Psi_{2}$ : $\mathbb{N} \rightarrow \mathbb{R}_{+}$, numbers $\Phi_{1}, \Phi_{2}, \Lambda \in \mathbb{R}_{+}$, positive integers $k_{0}, k_{1}$ with $k_{0}<k_{1}$, $m_{0} \in\{0, \ldots, m\}$ and polynomials $\left(Q_{k}\right)_{k_{0} \leq k \leq k_{1}}$, such that the following assumptions are satisfied:

(i) $\Phi_{2} \geq \Phi_{1} \geq c_{5}, \Lambda \geq \Psi_{1}(k+1) / \Psi_{2}(k) \geq 1$ for $k \in\left\{k_{0}, \ldots, k_{1}\right\}$,

(ii) $\Psi_{2}(k) \geq c_{5}\left(\log H\left(Q_{k}\right)+\operatorname{deg} Q_{k}\right)$ for $k \in\left\{k_{0}, \ldots, k_{1}\right\}$,

(iii) the polynomials $Q_{k} \in O_{K}\left[y_{1}, \ldots, y_{m}\right]\left(k_{0} \leq k \leq k_{1}\right)$ satisfy
(a) $\operatorname{deg} Q_{k} \leq \Phi_{1}$,
(b) $\log H\left(Q_{k}\right) \leq \Phi_{2}$,
(c) $\exp \left(-\Psi_{1}(k)\right) \leq\left|Q_{k}(\underline{\omega})\right| \leq \exp \left(-\Psi_{2}(k)\right)$,
(iv) $\Psi_{2}\left(k_{1}\right) \geq c_{5} \Lambda^{m_{0}-1} \Phi_{1}^{m_{0}-1} \max \left\{\Psi_{1}\left(k_{0}\right), \Phi_{2}\right\}$,

then

$$
\operatorname{trdeg}_{\mathbb{Q}} \mathbb{Q}(\underline{\omega}) \geq m_{0} .
$$

Proof. This is Theorem 1 in [T1] with slight modifications.

Lemma 4. Suppose $\underline{\omega} \in \mathbb{C}^{m}$. Then there exists a constant $c_{6}=c_{6}(\underline{\omega}, K)$ $\in \mathbb{R}_{+}$with the following property: If there exist functions $\Psi_{1}, \Psi_{2}: \mathbb{N}^{2} \rightarrow \mathbb{R}_{+}$, which are increasing in the first variable, numbers $\Phi_{1}, \Phi_{2}, \Lambda, U, \tau \in \mathbb{R}_{+}$, positive integers $N_{0}, N_{1}$ with $N_{0} \leq N_{1}$, for each $N \in\left\{N_{0}, \ldots, N_{1}\right\}$ positive integers $k_{0}(N), k_{1}(N)$ with $k_{0}(N) \leq k_{1}(N)$, and polynomials $Q_{k, N}$ for 
$N \in\left\{N_{0}, \ldots, N_{1}\right\}$ and $k \in\left\{k_{0}(N), \ldots, k_{1}(N)\right\}$, such that the following assumptions are satisfied for positive integers $D, H$ and all $N \in\left\{N_{0}, \ldots, N_{1}\right\}$, $k \in\left\{k_{0}(N), \ldots, k_{1}(N)\right\}:$

(i) (a) $\Phi_{2} \geq \Phi_{1} \geq c_{6}, \Lambda \geq \Psi_{1}(k+1, N) / \Psi_{2}(k, N) \geq 1$,

(b) $\Psi_{1}\left(k_{1}(N), N\right) \geq \Psi_{1}\left(k_{0}(N+1), N+1\right)$,

(c) $U \leq \max \left\{\Psi_{2}(k, N) \mid N_{0} \leq N \leq N_{1}, k_{0}(N) \leq k \leq k_{1}(N)\right\}$, $\tau \geq \min \left\{\Psi_{1}(k, N) \mid N_{0} \leq N \leq N_{1}, k_{0}(N) \leq k \leq k_{1}(N)\right\}$,

(ii) $\Psi_{2}(k, N) \geq c_{6}\left(\log H\left(Q_{k, N}\right)+\operatorname{deg} Q_{k, N}\right)$,

(iii) the polynomials $Q_{k, N} \in O_{K}\left[y_{1}, \ldots, y_{m}\right]$ satisfy

(a) $\operatorname{deg} Q_{k, N} \leq \Phi_{1}$,

(b) $\log H\left(Q_{k, N}\right) \leq \Phi_{2}$,

(c) $\exp \left(-\Psi_{1}(k, N)\right) \leq\left|Q_{k, N}(\underline{\omega})\right| \leq \exp \left(-\Psi_{2}(k, N)\right)$,

(iv) $U \geq c_{6} \Lambda^{m-1} \Phi_{1}^{m-1} \max \left\{\tau D, \Lambda\left(\Phi_{1} \log H+\Phi_{2} D\right)\right\}$,

then for all polynomials $R \in \mathbb{Z}\left[y_{1}, \ldots, y_{m}\right] \backslash\{0\}$ with $\operatorname{deg} R \leq D$, $H(R) \leq H$,

$$
|R(\underline{\omega})| \geq \exp (-U) .
$$

Pr o o f. Lemma 4 can be derived from Jabbouri's criterion $[\mathrm{J}]$ analogous to the proof of the proposition in [T2].

Lemma 5. Let $f_{1}, \ldots, f_{m} \in \mathbb{C}[[z]]$ be formal power series which satisfy

$$
A_{0}(z, f(z)) \underline{f}(T(z))=\underline{A}(z, \underline{f}(z)),
$$

where $f(z)=\left(f_{1}(z), \ldots, f_{m}(z)\right), T(z)=T_{1}(z) / T_{2}(z)$ is a rational function with $\bar{T}_{1}, T_{2} \in \mathbb{C}[z], d=\max \left\{\operatorname{deg} T_{1}, \operatorname{deg} T_{2}\right\}, \delta=\operatorname{ord}_{0} T \geq 2, \underline{A}(z, \underline{y})=$ $\left(A_{1}(z, \underline{y}), \ldots, A_{m}(z, \underline{y})\right)$, and $A_{i}(z, \underline{y}) \in \mathbb{C}\left[z, y_{1}, \ldots, y_{m}\right] \backslash\{0\}(0 \leq i \leq m)$ are polynomials with $\operatorname{deg}_{z} A_{i} \leq s$ and $\operatorname{deg}_{y_{1}, \ldots, y_{m}} A_{i} \leq t$. Suppose that $t^{m}<$ $\delta$ and $Q \in \mathbb{C}\left[z, y_{1}, \ldots, y_{m}\right]$ with $\operatorname{deg}_{z} Q \leq M, \operatorname{deg}_{y_{1}, \ldots, y_{m}} Q \leq N$ and $M \geq$ $N \geq 1$. If $Q(z, f(z)) \neq 0$, then

$$
\operatorname{ord}_{0} Q(z, \underline{f}(z)) \leq c_{7} M N^{m \log d /(\log \delta-m \log t)}
$$

with a constant $c_{7} \in \mathbb{R}_{+}$depending on $f$.

Proof. See Theorem 1 in [T3].

The following result of Kubota is often useful to verify the algebraic independence of the functions $f_{1}, \ldots, f_{m}$.

Lemma 6. Suppose $f_{i, j} \in \mathbb{C}[[z]](1 \leq i \leq m, 1 \leq j \leq n(i))$ are formal power series satisfying the functional equations

$$
f_{i, j}(z)=a_{i}(z) f_{i, j}(T(z))+b_{i, j}(z) \quad(1 \leq i \leq m, 1 \leq j \leq n(i))
$$

with $a_{i}, b_{i, j} \in \mathbb{C}(z), T \in \mathbb{C}(z)$ is not constant, $a_{i} \neq 0$, and $a_{i_{1}} / a_{i_{2}}$ is not of the form $g(T(z)) / g(z)$ with $g \in \mathbb{C}(z)$ for $i_{1} \neq i_{2}$. If $f_{1,1}, \ldots, f_{m, n(m)}$ are algebraically dependent, then there exist indices $1 \leq i_{1}<\ldots<i_{R} \leq m$, 
complex numbers $c_{i_{r}, j}$ for $1 \leq r \leq R$ and $1 \leq j \leq n\left(i_{r}\right)$, not all zero, and functions $g_{1}, \ldots, g_{R} \in \mathbb{C}(z)$ with the following properties:

(i) $g_{r}(z)=a_{i_{r}}(z) g_{r}(T(z))+\sum_{j=1}^{n\left(i_{r}\right)} c_{i_{r}, j} b_{i_{r}, j}(z)$ for $1 \leq r \leq R$,

(ii) there exist $m_{1}, \ldots, m_{R} \in \mathbb{Z}$, not all zero, such that

$$
\prod_{r=1}^{R}\left(\sum_{j=1}^{n\left(i_{r}\right)} c_{i_{r}, j} f_{i_{r}, j}(z)-g_{r}(z)\right)^{m_{r}} \in \mathbb{C}(z) .
$$

Proof. See Theorem 2 in [K2].

4. Proof of Theorem 1. The first step in the proof of the theorems is the reduction to the case $\omega=0$, as shown in [B3]. This is done by means of a suitable Möbius transformation $\Phi(z)$, which is defined as

$\Phi(z)= \begin{cases}z-\omega & \text { for } \omega \in \mathbb{C}, \\ \frac{1}{z-\beta} & \text { for } \omega=\infty \text { with an algebraic number } \beta \neq T^{k}(\alpha) \text { for } k \in \mathbb{N}_{0} .\end{cases}$

Then we consider the functions $f_{i}^{*}(z)=f_{i}\left(\Phi^{-1}(z)\right)$ and the transformation $T^{*}(z)=\Phi\left(T\left(\Phi^{-1}(z)\right)\right)$ (notice that $\operatorname{deg} T^{*}=\operatorname{deg} T$ and $\operatorname{ord}_{0} T^{*}=\operatorname{ord}_{\omega} T$ ). Since the functional equations

$$
a^{*}(z) \underline{f}^{*}(z)=A^{*}(z) \underline{f}^{*}\left(T^{*}(z)\right)+\underline{B}^{*}(z)
$$

with $a^{*}(z)=a\left(\Phi^{-1}(z)\right), A^{*}(z)=A\left(\Phi^{-1}(z)\right), \underline{B}^{*}(z)=\underline{B}\left(\Phi^{-1}(z)\right)$ hold, the assumptions of Theorem 1 are fulfilled for $f^{*}, d(z) a^{*}(z), d(z) A^{*}(z)$, $d(z) \underline{B}^{*}(z)$, where $d(z) \in \mathbb{Q}[z]$ is a common denominator for the rational functions in $A^{*}, \underline{B}^{*}, a^{*}$, and further $\omega=0$.

The next step in the proof of Theorem 1 is the estimate of the power series coefficients of the functions $f_{i}$ and the construction of an auxiliary function with high vanishing order at $z=0$. This yields a sequence of auxiliary polynomials in $f_{1}(\alpha), \ldots, f_{m}(\alpha)$. Application of Lemmas 3 and 5 and a suitable choice of the parameters completes the proof.

For the proof of Lemmas 7-9 we suppose that $T(z)=T_{1}(z) / T_{2}(z)$ with $T_{1}, T_{2} \in \overline{\mathbb{Q}}[z], \omega=0, d=\operatorname{deg} T \geq \delta=\operatorname{ord}_{0} T \geq 2$. Further we define for $f_{i}(z)=\sum_{h=0}^{\infty} f_{i, h} z^{h}$ the power series coefficients of the $j$ th power $f_{i}^{j}(z)$ by

$$
f_{i}^{j}(z)=\sum_{h=0}^{\infty}\left(\sum_{h_{1}+\ldots+h_{j}=h} f_{i, h_{1}} \ldots f_{i, h_{j}}\right) z^{h}=\sum_{h=0}^{\infty} f_{i, h}^{(j)} z^{h}
$$

and for $\underline{j}=\left(j_{1}, \ldots, j_{m}\right) \in \mathbb{N}_{0}^{m}$, 


$$
\begin{aligned}
\underline{f}(z)^{j} & =f_{1}^{j_{1}}(z) \ldots f_{m}^{j_{m}}(z) \\
& =\sum_{h=0}^{\infty}\left(\sum_{h_{1}+\ldots+h_{m}=h} f_{1, h_{1}}^{\left(j_{1}\right)} \ldots f_{m, h_{m}}^{\left(j_{m}\right)}\right) z^{h}=\sum_{h=0}^{\infty} f_{h}^{(j)} z^{h} .
\end{aligned}
$$

LEMMA 7. Suppose the above mentioned assumptions are fulfilled, and $f$ satisfies (3). Then for all $h \in \mathbb{N}_{0}$ and $j \in \mathbb{N}, j \in \mathbb{N}_{0}^{m}$ with $|j|=j_{1}+\ldots+j_{m}$,

(i) $f_{i, h} \in K$,

(ii) $\left|f_{i, h}\right| \leq \exp \left(c_{8}(1+h)\right), D^{\left[c_{8}(1+h)\right]} f_{i, h} \in O_{K}$,

(iii) $\left|f_{i, h}^{(j)}\right| \leq \exp \left(c_{9}(j+h)\right), D^{\left[c_{9}(j+h)\right]} f_{i, h}^{(j)} \in O_{K}$,

(iv) $\left|f_{h}^{(j)}\right| \leq \exp \left(c_{10}(|\underline{j}|+h)\right), D^{\left[c_{10}(|\underline{j}|+h)\right]} f_{h}^{(j)} \in O_{K}$,

where $D \in \mathbb{N}, c_{8}, c_{9}, c_{10} \in \mathbb{R}_{+}$, and the algebraic number field $K$ depend on $f_{1}, \ldots, f_{m}$.

Proof. Without loss of generality we may assume that $f_{i}(0)=0$ for all $i$ (otherwise we consider $f_{i}(z)-f_{i}(0)$ ), and the entries of $a(z)^{-1} A(z)$ (hence of $\left.a(z)^{-1} \underline{B}(z)\right)$ are regular in $z=0$. If there exist entries of $a(z)^{-1} A(z)$ which are not regular in $z=0$, and the pole order is at most $s$, we put

$$
R_{i}(z)=\sum_{h=0}^{s-1} f_{i, h} z^{h} \quad(1 \leq i \leq m), \quad \underline{R}(z)=\left(R_{1}(z), \ldots, R_{m}(z)\right),
$$

and consider the functions $g_{i}(z)=\left(f_{i}(z)-R_{i}(z)\right) z^{-s}$, which satisfy the functional equation

$$
\begin{aligned}
\underline{g}(z)= & T(z)^{s} z^{-s} a(z)^{-1} A(z) \underline{g}(T(z)) \\
& -z^{-s}\left(\underline{R}(z)-a(z)^{-1}(A(z) \underline{R}(T(z))+\underline{B}(z))\right),
\end{aligned}
$$

and then $T(z)^{s} z^{-s} a(z)^{-1} A(z)$ is regular in $z=0$ because of $\delta \geq 2$. Now let $K$ denote the algebraic number field which is generated by the coefficients of the power series expansion of the entries of $a(z)^{-1} A(z)$ and $a(z)^{-1} \underline{B}(z)$, the fixed point $\omega$ (remember the Möbius transformation $\Phi$ ), the coefficients of $T$, finitely many power series coefficients of $f_{1}, \ldots, f_{m}$ (if necessary, see above), and the point $\beta$ from the beginning of this section (if necessary). With $a(z)^{-1} A(z)=\left(a_{i, j}(z)\right)_{1 \leq i, j \leq m}, a(z)^{-1} \underline{B}(z)=\left(b_{i}(z)\right)_{1 \leq i \leq m}$ and

$$
\begin{gathered}
a_{i, j}(z)=\sum_{h=0}^{\infty} a_{i, j, h} z^{h}, \quad b_{i}(z)=\sum_{h=0}^{\infty} b_{i, h} z^{h}, \\
T(z)=\sum_{h=\delta}^{\infty} p_{h} z^{h}, \quad(T(z))^{l}=\sum_{h=\delta^{l}}^{\infty} p_{h}^{(l)} z^{h},
\end{gathered}
$$

the functional equation implies 


$$
\begin{aligned}
\sum_{h=1}^{\infty} f_{i, h} z^{h} & =\sum_{j=1}^{m}\left(\sum_{h=0}^{\infty} a_{i, j, h} z^{h}\right)\left(\sum_{l=1}^{\infty} f_{j, l}\left(\sum_{h=\delta^{l}}^{\infty} p_{h}^{(l)} z^{h}\right)\right)+\sum_{h=0}^{\infty} b_{i, h} z^{h} \\
& =\sum_{h=\delta}^{\infty}\left(\sum_{j=1}^{m} \sum_{k=\delta}^{h} a_{i, j, h-k}\left(\sum_{l=1}^{[\log k / \log \delta]} f_{j, l} p_{k}^{(l)}\right)\right) z^{h}+\sum_{h=0}^{\infty} b_{i, h} z^{h}
\end{aligned}
$$

and we get the identity

$$
f_{i, h}=\sum_{k=\delta}^{h} \sum_{j=1}^{m} a_{i, j, h-k}\left(\sum_{l=1}^{[\log k / \log \delta]} f_{j, l} p_{k}^{(l)}\right)+b_{i, h} .
$$

Now assertion (i) is obvious. According to Lemma 1(ii) the power series coefficients $p_{h}$ of $T$ are bounded by $\overline{p_{h}} \leq \exp \left(\gamma_{0}(h+1)\right)$ with $\gamma_{0} \in \mathbb{R}_{+}$, and then

$$
\left|p_{h}^{(l)}\right| \leq \sum_{h_{1}+\ldots+h_{l}=h}\left|p_{h_{1}} \ldots\right| p_{h_{l}} \leq \exp \left(\gamma_{1}(l+h)\right) .
$$

Together with (11) and the bounds of Lemma 1(ii) for the power series coefficients of the $a_{i, j}(z)$ and $b_{i}(z)$ this yields the first part of (ii) by induction, and with suitable $D \in \mathbb{N}$ the second part of (ii) follows from Lemma 1(iii).

Assertions (iii) and (iv) are consequences of (ii) and the identities (9), (10) (notice that the number of $\underline{h} \in \mathbb{N}_{0}^{j}$ with $|\underline{h}|=h$ is bounded by $\left(\begin{array}{c}h+j-1 \\ j-1\end{array}\right) \leq$ $\left.2^{h+j}\right)$

Lemma 8. For $N \in \mathbb{N}$ there exists a polynomial $R_{N}(z, \underline{y}) \in O_{K}\left[z, y_{1}, \ldots\right.$ $\left.\ldots, y_{m}\right] \backslash\{0\}$ with the following properties:

(i) $\operatorname{deg}_{z} R_{N} \leq N, \operatorname{deg}_{y} R_{N} \leq N$,

(ii) $H\left(R_{N}\right) \leq \exp \left(c_{11} \bar{N}^{1+m}\right)$,

(iii) $c_{12} N^{1+m} \leq \nu(N)=\operatorname{ord}_{0} R_{N}(z, \underline{f}(z)) \leq c_{13} N^{1+m \log d / \log \delta}$.

Proof. Put

$$
R_{N}(z, \underline{y})=\sum_{\nu=0}^{N} \sum_{|\underline{\mu}| \leq N} r_{\nu, \underline{\mu}} z^{\nu} \underline{y}^{\underline{\mu}}
$$

with unknown coefficients $r_{\nu, \underline{\mu}}$. Then

$$
R_{N}(z, \underline{f}(z))=\sum_{\nu=0}^{N} \sum_{|\underline{\mu}| \leq N} r_{\nu, \underline{\mu}} z^{\nu} \underline{f}(z) \underline{\mu}=\sum_{h=0}^{\infty} \beta_{h} z^{h}
$$

with

$$
\beta_{h}=\sum_{\nu=0}^{\min \{h, N\}} \sum_{|\underline{\mu}| \leq N} r_{\nu, \underline{\mu}} f_{h-\nu}^{(\underline{\mu})}
$$


The left-hand inequality of assertion (iii) is equivalent to the condition $\beta_{h}=$ 0 for $0 \leq h<c_{12} N^{1+m}$. This yields at most $\left[c_{12} N^{1+m}\right]+1$ linear equations in the $(N+1)\left(\begin{array}{c}N+m \\ m\end{array}\right)$ unknowns $r_{\nu, \mu}$. After multiplication with $D^{\left[c_{12} N^{1+m}\right]}$ (see Lemma 7) the coefficients of the linear equations are algebraic integers, and the houses are bounded by $\exp \left(\gamma_{0} N^{1+m}\right)$. Since $(N+1)\left(\begin{array}{c}N+m \\ m\end{array}\right) \geq \frac{1}{m !} N^{1+m}>$ $2 c_{12} N^{1+m}+1$ for suitable $c_{12} \in \mathbb{R}_{+}$, Siegel's lemma yields the assertion of Lemma 8 apart from the upper bound for the zero order $\nu(N)$ in (iii), but this is a consequence of Lemma 5 .

Lemma 9. For $k \in \mathbb{N}$ with $\delta^{k} \geq c_{14} \nu(N)$,

$$
\exp \left(-c_{15} \nu(N) \delta^{k}\right) \leq\left|R_{N}\left(T^{k}(\alpha), \underline{f}\left(T^{k}(\alpha)\right)\right)\right| \leq \exp \left(-c_{16} \nu(N) \delta^{k}\right),
$$

where the constants $c_{14}, c_{15}, c_{16} \in \mathbb{R}_{+}$depend only on $\underline{f}$ and $\alpha$.

Proof. From Lemma 7 and (12) we get (notice that $h \geq c_{12} N^{1+m}$ )

$$
\left|\beta_{h}\right| \leq \mid \beta_{h} \leq \exp \left(\gamma_{0} h\right), \quad D^{\left[\gamma_{0} h\right]} \beta_{h} \in O_{K} .
$$

Then we consider

$$
R_{N}\left(T^{k}(\alpha), \underline{f}\left(T^{k}(\alpha)\right)\right)=\beta_{\nu(N)}\left(T^{k}(\alpha)\right)^{\nu(N)}\left(1+\sum_{h=1}^{\infty} \frac{\beta_{h+\nu(N)}}{\beta_{\nu(N)}}\left(T^{k}(\alpha)\right)^{h}\right) .
$$

Since

$$
\left.\left|\beta_{\nu(N)}\right| \geq\left(D^{\left[\gamma_{0} \nu(N)\right]} \sqrt{\beta_{\nu(N)}}\right)\right)^{-[K: \mathbb{Q}]}
$$

and

$$
\left|\frac{\beta_{h+\nu(N)}}{\beta_{\nu(N)}}\right| \leq \exp \left(\gamma_{1}(h+\nu(N))\right)
$$

for $h \in \mathbb{N}$, Lemma 2 implies for $k \in \mathbb{N}$ with $\delta^{k} \geq \gamma_{2} \nu(N)$,

$$
\left|\sum_{h=1}^{\infty} \frac{\beta_{h+\nu(N)}}{\beta_{\nu(N)}}\left(T^{k}(\alpha)\right)^{h}\right| \leq \sum_{h=1}^{\infty} \exp \left(\gamma_{1}(h+\nu(N))-\gamma_{3} h \delta^{k}\right)<\frac{1}{2},
$$

hence

$$
\frac{1}{2}\left|\beta_{\nu(N)}\right|\left|T^{k}(\alpha)\right|^{\nu(N)} \leq\left|R_{N}\left(T^{k}(\alpha), \underline{f}\left(T^{k}(\alpha)\right)\right)\right| \leq \frac{3}{2}\left|\beta_{\nu(N)}\right|\left|T^{k}(\alpha)\right|^{\nu(N)} .
$$

Now (13), (14) together with Lemma 2 complete the proof.

From now on we suppose in addition that $\delta=\operatorname{ord}_{0} T=\operatorname{deg} T=d$, i.e. the assumptions of Theorem 1 are fulfilled with $\omega=0$. For the application of Lemma 4 we define polynomials $R_{k, N} \in K[z, \underline{y}]$ for $k, N \in \mathbb{N}$ with $\delta^{k} \geq$ $c_{14} \nu(N)$ by

$$
\begin{aligned}
R_{0, N}(z, \underline{y}) & =R_{N}(z, \underline{y}), \\
R_{k+1, N}(z, \underline{y}) & =(\operatorname{det} A(z))^{N} T_{2}(z)^{d_{k} N} R_{k, N}\left(T(z), A(z)^{-1}(a(z) \underline{y}-\underline{B}(z))\right),
\end{aligned}
$$


where the degree of the entries of $A(z)$ and $\underline{B}(z)$ is at most $s \in \mathbb{N}$, and $d_{k}=c_{17}\left(d^{k}-1\right) /(d-1)+d^{k}$ with $c_{17}=m s$.

Lemma 10. Suppose $k, N \in \mathbb{N}$. Then

(i) $R_{k, N} \in K[z, \underline{y}]$,

(ii) $\operatorname{deg}_{z} R_{k, N} \leq d_{k} N \leq 2 c_{17} d^{k} N, \operatorname{deg}_{y} R_{k, N} \leq N$,

(iii) $H\left(R_{k, N}\right) \leq \exp \left(c_{18} N\left(d^{k}+N^{m}\right)\right)$,

and if $d^{k} \geq c_{19} \nu(N)$, then

(iv) $\exp \left(-c_{20} \nu(N) d^{k}\right) \leq\left|R_{k, N}(\alpha, \underline{f}(\alpha))\right| \leq \exp \left(-c_{21} \nu(N) d^{k}\right)$.

Proof. (i), (ii) are proved by induction; (i) follows from the fact that the matrix $\operatorname{det} A(z) A(z)^{-1}$ has entries in $K[z]$, and (ii) is a consequence of $\operatorname{deg} T=d$ and the definition of $c_{17}$. Suppose that $L$ is an upper bound for the length of $a(z)$ and the entries of $A(z)$ and $\underline{B}(z)$. Then assertion (iii) follows from

$$
\begin{aligned}
H\left(R_{k+1, N}\right) & \leq L\left(R_{k+1, N}\right) \\
& \leq L\left(R_{k, N}\right) \max \{1, L\}^{m N} \max \left\{1, L\left(T_{1}\right), L\left(T_{2}\right)\right\}^{d_{k} N} \\
& \leq L\left(R_{N}\right) \exp \left(\gamma_{0} \sum_{l=0}^{k} d_{l} N\right) \leq \exp \left(\gamma_{1} d^{k+1} N+\gamma_{2} N^{1+m}\right) .
\end{aligned}
$$

The last assertion is a consequence of $d=\delta$, Lemma 8 , and

$$
\begin{aligned}
& R_{k, N}(\alpha, \underline{f}(\alpha)) \\
& =\prod_{j=0}^{k-1}\left(\operatorname{det} A\left(T^{j}(\alpha)\right)\right)^{N} \prod_{j=0}^{k-1}\left(T_{2}\left(T^{j}(\alpha)\right)\right)^{d_{k-1-j} N} R_{N}\left(T^{k}(\alpha), \underline{f}\left(T^{k}(\alpha)\right)\right),
\end{aligned}
$$

since

$$
\exp \left(-\gamma_{3} d^{k} N\right) \leq \prod_{j=0}^{k-1}\left|\operatorname{det} A\left(T^{j}(\alpha)\right)\right|^{N} \leq \exp \left(\gamma_{4} d^{k} N\right)
$$

and

$$
\exp \left(-\gamma_{5} d^{k} N\right) \leq \prod_{j=0}^{k-1}\left|T_{2}\left(T^{j}(\alpha)\right)\right|^{d_{k-1-j} N} \leq \exp \left(\gamma_{6} d^{k} N\right)
$$

Suppose that $D_{1}$ is a denominator of $\alpha, D_{2}$ is a common denominator of the coefficients of $T(z)$, and $D_{3}$ is a common denominator of the coefficients of $a(z)$ and the entries of $A(z)$ and $\underline{B}(z)$. Then we put

$$
Q_{k, N}(\underline{y})=\left(D_{1} D_{2}\right)^{\left[2 c_{17} d^{k} N\right]+1} D_{3}^{m k N} R_{k, N}(\alpha, \underline{y}) .
$$


Thus for $N \geq N_{0}$ and $k \in \mathbb{N}$ with $d^{k} \geq c_{22} N^{1+m}$ (cf. Lemma 8(iii)),

$$
\begin{gathered}
Q_{k, N} \in O_{K}[\underline{y}], \quad \operatorname{deg} Q_{k, N} \leq N, \quad H\left(Q_{k, N}\right) \leq \exp \left(c_{23} d^{k} N\right), \\
\exp \left(-c_{24} d^{k} N^{1+m}\right) \leq\left|Q_{k, N}(\underline{f}(\alpha))\right| \leq \exp \left(-c_{25} d^{k} N^{1+m}\right) .
\end{gathered}
$$

With sufficiently large constants $\gamma_{0}, \gamma_{1} \in \mathbb{R}_{+}$, which depend only on $f, \alpha, N_{0}$, and the constant $c_{6}$ of Lemma 4 , we choose $N_{1}=\left[\gamma_{0} D\right]$ and the parameters $k_{0}(N), k_{1}(N)$ for $N \in\left\{N_{0}, \ldots, N_{1}\right\}$ such that

$$
\begin{gathered}
d^{k_{0}(N)-1}<c_{22} N^{1+m} \leq d^{k_{0}(N)}, \\
k_{1}=k_{1}(N)=\left[\frac{1}{\log d} \log \left(D^{m+1}+\frac{\log H}{D}\right)+\gamma_{1}\right],
\end{gathered}
$$

$D$ and $H$ as in the assumptions of Theorem 1. Hence $k_{0}(N) \leq k_{1}$, and for the application of Lemma 4 we define

$$
\begin{gathered}
\Phi_{1}=N_{1}, \quad \Phi_{2}=c_{23} N_{1} d^{k_{1}} \\
\Psi_{1}(k, N)=c_{24} d^{k} N^{1+m}, \quad \Psi_{2}(k, N)=c_{25} d^{k} N^{1+m} .
\end{gathered}
$$

Then obviously (i), (ii), (iii) of Lemma 4 are fulfilled with $\Lambda=d c_{24} / c_{25}$ and

$$
U=c_{24} d^{k_{1}} N_{1}^{1+m}, \quad \tau=c_{24} d^{k_{0}\left(N_{0}\right)} N_{0}^{1+m} .
$$

Furthermore, we see that

$$
\begin{aligned}
U & \geq \gamma_{2} N_{1}^{m} \max \left\{\log H+d^{k_{1}} D, \tau D / N_{1}\right\} \\
& \geq c_{6} \Lambda^{m-1} \Phi_{1}^{m-1} \max \left\{\tau D, \Lambda\left(\Phi_{1} \log H+\Phi_{2} D\right)\right\},
\end{aligned}
$$

and Lemma 4 implies

$$
\begin{aligned}
|Q(\underline{f}(\alpha))| & >\exp (-U) \\
& \geq \exp \left(-\gamma_{3} d^{k_{1}} N_{1}^{1+m}\right) \\
& \geq \exp \left(-\gamma_{4} D^{m+1}\left(D^{m+1}+\frac{\log H}{D}\right)\right) .
\end{aligned}
$$

5. Proof of Theorem 2. The first part of the proof up to Lemma 9 and the definition of the polynomials $R_{k, N}$ in the paragraph after Lemma 9 is identical with the proof of Theorem 1 . Since $2 \leq \delta \leq d$, Lemma 10 must be slightly modified.

Lemma 11. Suppose $k, N \in \mathbb{N}$. Then

(i) $R_{k, N} \in K[z, \underline{y}]$,

(ii) $\operatorname{deg}_{z} R_{k, N} \leq \bar{d}_{k} N \leq 2 c_{17} d^{k} N, \operatorname{deg}_{\underline{y}} R_{k, N} \leq N$,

(iii) $H\left(R_{k, N}\right) \leq \exp \left(c_{18} N\left(d^{k}+N^{m}\right)\right)$,

and if $\delta^{k} \geq c_{26} \nu(N)$ and $N d^{k} \leq c_{27} \nu(N) \delta^{k}$, then

(iv) $\exp \left(-c_{28} \nu(N) \delta^{k}\right) \leq\left|R_{k, N}(\alpha, \underline{f}(\alpha))\right| \leq \exp \left(-c_{29} \nu(N) \delta^{k}\right)$. 
Proof. The additional assumption in (iv) is necessary to compensate the bounds of Lemma 9 and (15), (16).

With denominators $D_{1}, D_{2}, D_{3}$ as in (17) we define polynomials $Q_{k, N}$ by

$$
Q_{k, N}(\underline{y})=\left(D_{1} D_{2}\right)^{\left[2 c_{17} d^{k} N\right]+1} D_{3}^{m k N} R_{k, N}(\alpha, \underline{y}) \text {. }
$$

Thus for $k \in \mathbb{N}$ with $N d^{k} \leq c_{30} \nu(N) \delta^{k}$ and $\delta^{k} \geq c_{31} \nu(N)$ we have

$$
\begin{gathered}
Q_{k, N} \in O_{K}[\underline{y}], \quad \operatorname{deg} Q_{k, N} \leq N, \quad H\left(Q_{k, N}\right) \leq \exp \left(c_{32} d^{k} N\right), \\
\exp \left(-c_{33} \delta^{k} \nu(N)\right) \leq\left|Q_{k, N}(\underline{f}(\alpha))\right| \leq \exp \left(-c_{34} \delta^{k} \nu(N)\right) .
\end{gathered}
$$

With sufficiently large $\gamma_{0}, \gamma_{1} \in \mathbb{R}_{+}$, which depend on $\underline{f}$ and $\alpha$, we define

$$
k_{0}=\left[\frac{\log \nu(N)}{\log \delta}+\gamma_{0}\right], \quad k_{1}=\left[\frac{\log \nu(N)-m_{0} \log N}{\log d-\log \delta}-\gamma_{1}\right]
$$

(notice that $c_{30} \in \mathbb{R}_{+}$may be very small). Then obviously $N d^{k} \leq c_{30} \nu(N) \delta^{k}$ and $\delta^{k} \geq c_{31} \nu(N)$ for $k_{0} \leq k \leq k_{1}$ (without loss of generality $m_{0} \geq 1$ ), and $k_{0} \leq k_{1}$ is shown in (19). Furthermore,

$$
\nu(N) \delta^{k_{1}} \geq \gamma_{2} N^{m_{0}} d^{k_{1}}
$$

and the definition of $m_{0}, k_{0}, k_{1}$ together with $\nu(N) \geq c_{12} N^{1+m}$ yields

$$
\delta^{k_{1}} \geq \gamma_{3} N^{m_{0}-1} \delta^{k_{0}}
$$

with $\gamma_{2}, \gamma_{3} \in \mathbb{R}_{+}$for $N \geq N_{0}\left(\gamma_{0}, \ldots, \gamma_{3}\right)$. Thus we define

$$
\begin{array}{r}
\Phi_{1}=N, \quad \Phi_{2}=c_{32} d^{k_{1}} N, \\
\Psi_{1}(k)=c_{33} \delta^{k} \nu(N), \quad \Psi_{2}(k)=c_{34} \delta^{k} \nu(N), \quad \Lambda=\delta c_{33} / c_{34},
\end{array}
$$

and if we now fix $N \in \mathbb{N}$ sufficiently large with respect to $\gamma_{0}, \ldots, \gamma_{3}, \delta, f, \alpha$, and $c_{5}$, we put $Q_{k}=Q_{k, N}$ for $k_{0} \leq k \leq k_{1}$ and this value of $N$. Then (18), (19) imply

$$
\Psi_{2}(k) \geq c_{5} \Lambda^{m_{0}-1} \Phi_{1}^{m_{0}-1} \max \left\{\Psi_{1}\left(k_{0}\right), \Phi_{2}\right\},
$$

and the other assumptions of Lemma 3 are also fulfilled for this choice of parameters. The application of Lemma 3 completes the proof of Theorem 2.

6. Proof of Theorem 3. Under the assumptions of Theorem 3 we can give sharper bounds for the power series coefficients of $f_{1}, \ldots, f_{m}$ in the expansion at $\omega$. This yields a weaker condition for $k_{0}$, hence a better bound for $m_{0}$.

Analogously to Section 4 we apply the Möbius transformation $\Phi$ to get $\omega=0$. Then the sharper estimates for the power series coefficients depend on the fact that $a(z)=1$, and $T(z)$ and the entries of $A(z)$ and $\underline{B}(z)$ are polynomials. For the sake of simplicity the case $\omega=\infty$ is excluded, because then $\Phi$ transforms the functional equation into another system, where in general $a(z)$ is not constant, and $T(z)$ is rational. 
Since the proof of Theorem 3 is analogous to the proof of Theorem 2 apart from the estimates for the power series coefficients, most proofs are shortened or omitted.

Lemma 12. Suppose that the assumptions of Theorem 3 are fulfilled with $\omega=0$. Then for all $h \in \mathbb{N}_{0}$ and $j \in \mathbb{N}, \underline{j} \in \mathbb{N}_{0}^{m}$,

(i) $f_{i, h} \in K$,

(ii) $\sqrt[f_{i, h}]{ } \leq \exp \left(c_{34} \log (h+2)\right), D^{\left[c_{34} \log (h+2)\right]} f_{i, h} \in O_{K}$,

(iii) $f_{i, h}^{(j)} \leq \exp \left(c_{35} j \log (h+2)\right), D^{\left[c_{35} j \log (h+2)\right]} f_{i, h}^{(j)} \in O_{K}$,

(iv) $\left|f_{h}^{(j)}\right| \leq \exp \left(c_{36}|\underline{j}| \log (h+2)\right), D^{\left[c_{36}|\underline{j}| \log (h+2)\right]} f_{h}^{(j)} \in O_{K}$,

where $D \in \mathbb{N}, c_{34}, c_{35}, c_{36} \in \mathbb{R}_{+}$, and the algebraic number field $K$ depend on $\underline{f}$.

Proof. Without loss of generality $f_{i}(0)=0$ for all $i$ (since $f_{1}(0), \ldots$ $\ldots, f_{m}(0) \in \overline{\mathbb{Q}}$, the functions $f_{i}(z)-f_{i}(0), 1 \leq i \leq m$, satisfy functional equations of the required form). Then with $A(z)=\left(a_{i, j}(z)\right)_{1 \leq i, j \leq m}, \underline{B}(z)=$ $\left(B_{i}(z)\right)_{1 \leq i \leq m}$ and

$$
\begin{gathered}
a_{i, j}(z)=\sum_{h=0}^{s} a_{i, j, h} z^{h}, \quad B_{i}(z)=\sum_{h=0}^{s} b_{i, h} z^{h}, \\
T(z)=\sum_{h=\delta}^{d} p_{h} z^{h}, \quad(T(z))^{l}=\sum_{h=\delta^{l}}^{d^{l}} p_{h}^{(l)} z^{h},
\end{gathered}
$$

the functional equation implies

$$
\begin{aligned}
\sum_{h=1}^{\infty} f_{i, h} z^{h}= & \sum_{j=1}^{m}\left(\sum_{h=0}^{s} a_{i, j, h} z^{h}\right)\left(\sum_{l=1}^{\infty} f_{j, l}\left(\sum_{h=\delta^{l}}^{d^{l}} p_{h}^{(l)} z^{h}\right)\right)+\sum_{h=0}^{s} b_{i, h} z^{h} \\
= & \sum_{h=\delta}^{\infty}\left(\sum_{j=1}^{m} \sum_{k=\max \{\delta, h-s\}}^{h} a_{i, j, h-k}\left(\sum_{\log k / \log d \leq l \leq \log k / \log \delta} f_{j, l} p_{k}^{(l)}\right)\right) z^{h} \\
& +\sum_{h=0}^{s} b_{i, h} z^{h},
\end{aligned}
$$

and from the identity

$$
\text { (20) } f_{i, h}=\sum_{k=\max \{\delta, h-s\}}^{h} \sum_{j=1}^{m} a_{i, j, h-k}\left(\sum_{\log k / \log d \leq l \leq \log k / \log \delta} f_{j, l} p_{k}^{(l)}\right)+b_{i, h}
$$

(with $b_{i, h}=0$ for $h>s$ ) assertion (i) follows immediately. Since

$$
\left|p_{h}^{(l)}\right| \leq \sum_{h_{1}+\ldots+h_{l}=h}\left|p_{h_{1}} \ldots\right| p_{h_{l}} \leq \exp \left(\gamma_{0} l\right)
$$


(notice that $\delta \leq h_{i} \leq d$ for $i=1, \ldots, l$ ), the first part of (ii) follows from (20), if we choose $D \in \mathbb{N}$ as a suitable denominator for the coefficients of $T(z)$ and the entries of $A(z)$ and $\underline{B}(z)$. Then (iii), (iv) can be derived from (9), (10) respectively (notice that the number of $\underline{h} \in \mathbb{N}_{0}^{j}$ with $|\underline{h}|=h$ is bounded by $\left(\begin{array}{c}h+j-1 \\ j-1\end{array}\right) \leq \exp (j \log (h+1))$.

Lemma 13. For $N \in \mathbb{N}$ there exists a polynomial $R_{N}(z, y) \in O_{K}\left[z, y_{1}, \ldots\right.$ $\left.\ldots, y_{m}\right] \backslash\{0\}$ with the following properties:

(i) $\operatorname{deg}_{z} R_{N} \leq N, \operatorname{deg}_{\underline{y}} R_{N} \leq N$,

(ii) $H\left(R_{N}\right) \leq \exp \left(c_{37} \bar{N} \log (N+1)\right)$,

(iii) $c_{38} N^{1+m} \leq \nu(N)=\operatorname{ord}_{0} R_{N}(z, \underline{f}(z))$.

Proof. Analogous to Lemma 8.

Lemma 14. For $k \in \mathbb{N}$ with $\delta^{k} \geq c_{39} N \log \nu(N)$,

$$
\exp \left(-c_{40} \nu(N) \delta^{k}\right) \leq\left|R_{N}\left(T^{k}(\alpha), \underline{f}\left(T^{k}(\alpha)\right)\right)\right| \leq \exp \left(-c_{41} \nu(N) \delta^{k}\right),
$$

where $c_{39}, c_{40}, c_{41} \in \mathbb{R}_{+}$depend only on $\underline{f}$ and $\alpha$.

Pro of. Analogous to Lemma 9. Notice that

$$
\left|\beta_{h}\right| \leq \mid \beta_{h} \leq \exp \left(\gamma_{0} N \log h\right), \quad D^{\left[\gamma_{0} N \log h\right]} \beta_{h} \in O_{K}
$$

and $h \geq \nu(N)$.

Now we define polynomials $R_{k, N}$ by

$$
\begin{aligned}
R_{0, N}(z, \underline{y}) & =R_{N}(z, \underline{y}), \\
R_{k+1, N}(z, \underline{y}) & =(\operatorname{det} A(z))^{N} R_{k, N}\left(T(z), A(z)^{-1}(\underline{y}-\underline{B}(z))\right),
\end{aligned}
$$

where the degree of the entries of $A(z)$ and $\underline{B}(z)$ is at most $s$.

Lemma 15. Suppose $k, N \in \mathbb{N}$. Then

(i) $R_{k, N} \in K[z, \underline{y}]$,

(ii) $\operatorname{deg}_{z} R_{k, N} \leq c_{42}\left(d^{k}-1\right) /(d-1)+d^{k} \leq 2 c_{42} d^{k}$, $\operatorname{deg}_{y} R_{k, N} \leq N$,

(iii) $H\left(R_{k, N}\right) \leq \exp \left(c_{43} N\left(\log (N+1)+d^{k}\right)\right)$

with $c_{42}=s m, c_{43} \in \mathbb{R}_{+}$.

If $\delta^{k} \geq c_{44} N \log \nu(N)$ and $N d^{k} \leq c_{45} \nu(N) \delta^{k}$, then

(iv) $\exp \left(-c_{46} \nu(N) \delta^{k}\right) \leq\left|R_{k, N}(\alpha, \underline{f}(\alpha))\right| \leq \exp \left(-c_{47} \nu(N) \delta^{k}\right)$.

Pro of. Analogous to Lemma 10 resp. Lemma 11.

Suppose that $D_{1}$ is a denominator of $\alpha, D_{2}$ is a common denominator of the coefficients of $T(z)$, and $D_{3}$ is a common denominator of the coefficients of the entries of $A(z)$ and $\underline{B}(z)$. Then we define

$$
Q_{k, N}(\underline{y})=\left(D_{1} D_{2}\right)^{\left[2 c_{42} d^{k} N\right]+1} D_{3}^{m k N} R_{k, N}(\alpha, \underline{y}) .
$$


Thus for $N \geq N_{0}$ and $\delta^{k} \geq c_{48} N \log \nu(N)$ and $N d^{k} \leq c_{49} \nu(N) \delta^{k}$ we have

$$
\begin{gathered}
Q_{k, N} \in O_{K}[\underline{y}], \quad \operatorname{deg} Q_{k, N} \leq N, \quad H\left(Q_{k, N}\right) \leq \exp \left(c_{50} d^{k} N\right), \\
\exp \left(-c_{51} \delta^{k} \nu(N)\right) \leq\left|Q_{k, N}(\underline{f}(\alpha))\right| \leq \exp \left(-c_{52} \delta^{k} \nu(N)\right) .
\end{gathered}
$$

With sufficiently large $\gamma_{0}, \gamma_{1} \in \mathbb{R}_{+}$, which depend on $\underline{f}$ and $\alpha$, we choose

$$
k_{0}=\left[\frac{\log (N \log \nu(N))}{\log \delta}+\gamma_{0}\right], \quad k_{1}=\left[\frac{\log \nu(N)-m_{0} \log N}{\log d-\log \delta}-\gamma_{1}\right] .
$$

This implies $\delta^{k} \geq c_{48} N \log \nu(N)$ and $N d^{k} \leq c_{49} \nu(N) \delta^{k}$. Furthermore,

$$
\nu(N) \delta^{k_{1}} \geq \gamma_{2} N^{m_{0}} d^{k_{1}}
$$

for $N \geq N_{0}\left(\gamma_{2}\right)$. Since $m_{0} \log d<(1-\varepsilon)(m+1) \log \delta$ for some $\varepsilon \in \mathbb{R}_{+}$and $\nu(N) \geq c_{38} N^{1+m}$, we have for all $N \geq N_{0}\left(\gamma_{0}, \ldots, \gamma_{3}, \varepsilon\right)$,

$$
\delta^{k_{1}} \geq \gamma_{3} N^{m_{0}-1} \delta^{k_{0}} \text {. }
$$

Thus let

$$
\begin{gathered}
\Phi_{1}=N, \quad \Phi_{2}=c_{50} N d^{k_{1}} \\
\Psi_{1}(k)=c_{51} \delta^{k} \nu(N), \quad \Psi_{2}(k)=c_{52} \delta^{k} \nu(N), \quad \Lambda=\delta c_{51} / c_{52},
\end{gathered}
$$

where $N$ is fixed sufficiently large with respect to $\gamma_{0}, \ldots, \gamma_{3}, \varepsilon, \delta, f, \alpha$, and $c_{5}$, and put

$$
Q_{k}(\underline{y})=Q_{k, N}(\underline{y})
$$

for $k_{0} \leq k \leq k_{1}$ and this value of $N$. Then

$$
\Psi_{2}\left(k_{1}\right) \geq c_{5} \Lambda^{m_{0}-1} \Phi_{1}^{m_{0}-1} \max \left\{\Psi_{1}\left(k_{0}\right), \Phi_{2}\right\},
$$

and since all other assumptions of Lemma 3 are fulfilled, the assertion of Theorem 3 now follows from Lemma 3.

\section{References}

[B1] P.-G. Becker, Effective measures for algebraic independence of the values of Mahler type functions, Acta Arith. 58 (1991), 239-250.

[B2] - , Algebraic independence of the values of certain series by Mahler's method, Monatsh. Math. 114 (1992), 183-198.

[B3] - , Transcendence of the values of functions satisfying generalized Mahler type functional equations, J. Reine Angew. Math. 440 (1993), 111-128.

[B4] - , Transcendence measures for the values of generalized Mahler functions in arbitrary characteristic, Publ. Math. Debrecen, to appear.

[BB] P.-G. Becker and W. Bergweiler, Transcendency of local conjugacies in complex dynamics and transcendency of their values, Manuscripta Math. 81 (1993), 329337.

[DS] J. L. Davison and J. E. Shallit, Continued fractions for some alternating series, Monatsh. Math. 111 (1991), 119-126. 
[J] E. M. Jabbouri, Sur un critère pour l'indépendance algébrique de P. Philippon, in: Approximations Diophantiennes et Nombres Transcendants, P. Philippon (ed.), W. de Gruyter, Berlin, 1992, 195-202.

[K1] K. K. Kubota, Linear functional equations and algebraic independence, in: Transcendence Theory: Advances and Applications, A. Baker and D. W. Masser (eds.), Academic Press, New York, 1977, 227-229.

[K2] - On the algebraic independence of holomorphic solutions of certain functional equations and their values, Math. Ann. 227 (1977), 9-50.

[L] J. H. Loxton, Automata and transcendence, in: New Advances in Transcendence Theory, A. Baker (ed.), Cambridge University Press, Cambridge, 1988, 215-228.

[LP] J. H. Loxton and A. J. van der Poorten, Transcendence and algebraic independence by a method of Mahler, in: Transcendence Theory: Advances and Applications, A. Baker and D. W. Masser (eds.), Academic Press, New York, 1977, 211-226.

[M1] K. Mahler, Arithmetische Eigenschaften der Lösungen einer Klasse von Funktionalgleichungen, Math. Ann. 101 (1929), 342-366.

[M2] -, Über das Verschwinden von Potenzreihen mehrerer Veränderlichen in speziellen Punktfolgen, ibid. 103 (1930), 573-587.

[M3] - , Arithmetische Eigenschaften einer Klasse transzendental-transzendenter Funktionen, Math. Z. 32 (1930), 545-585.

[M4] -, Remarks on a paper by W. Schwarz, J. Number Theory 1 (1969), 512-521.

[Ni1] K. Nishioka, On a problem of Mahler for transcendency of function values, J. Austral. Math. Soc. Ser. A 33 (1982), 386-393.

[Ni2] - Algebraic independence measures of the values of Mahler functions, J. Reine Angew. Math. 420 (1991), 203-214.

[NT] K. Nishioka and T. Töpfer, Transcendence measures and nonlinear functional equations of Mahler type, Arch. Math. (Basel) 57 (1991), 370-378.

[Ta] J. Tamura, Symmetric continued fractions related to certain series, J. Number Theory 38 (1991), 251-264.

[T1] T. Töpfer, An axiomatization of Nesterenko's method and applications on Mahler functions, ibid. 49 (1994), 1-26.

[T2] - , An axiomatization of Nesterenko's method and applications on Mahler functions II, Compositio Math., to appear.

[T3] - Zero order estimates for functions satisfying generalized functional equations of Mahler type, Acta Arith., to appear.

MATHEMATISCHES INSTITUT

UNIVERSITÄT ZU KÖLN

WEYERTAL 86-90

D-50931 KÖLN, GERMANY 